\title{
Metastatic urachal cancer treated effectively with gemcitabine/cisplatin combination chemotherapy and radiotherapy: A case report
}

\author{
HIROSHI YAEGASHI, SUGURU KADOMOTO, RENATO NAITO, TOMOYUKI MAKINO, \\ HIROAKI IWAMOTO, TAKAHIRO NOHARA, KAZUYOSHI SHIGEHARA, \\ KOUJI IZUMI, YOSHIFUMI KADONO and ATSUSHI MIZOKAMI
}

\author{
Department of Integrative Cancer Therapy and Urology, Kanazawa University Graduate \\ School of Medical Science, Kanazawa, Ishikawa 920-8640, Japan
}

Received December 13, 2018; Accepted May 16, 2019

DOI: $10.3892 / \mathrm{mco} .2019 .1865$

\begin{abstract}
Urachal cancer often presents at an advanced stage with poor prognosis due to the lack of an effective systematic therapeutic strategy. We experienced a case of metastatic urachal cancer treated effectively by combination chemotherapy and radiotherapy. A 55-year-old female presented to our department with right lower abdominal pain. A transurethral biopsy of an urachal tumor suggested urachal adenocarcinoma. The patient underwent chemotherapy with gemcitabine and cisplatin for metastatic urachal cancer. As tumor markers declined and the radiological findings indicated stability of disease, external beam radiotherapy was then administered to the primary site. Chemotherapy was then administered again in response to tumor markers gradually increasing and the progression of multiple peritoneal metastases. However, the patient did not complete chemotherapy due to hematological toxicity. The patient succumbed to primary disease 23 months after initial diagnosis. Previous studies have reported that the median time from the diagnosis of metastatic urachal cancer to mortality is just over 1 year. By contrast, in the present case the patient survived up to 2 years with combination chemotherapy and radiotherapy, a rare incidence worthy of reporting.
\end{abstract}

\section{Introduction}

Urachal cancer (UrC) is a very rare but highly malignant tumor with an incidence of $<1 \%$ of all bladder cancers (1-3).

Correspondence to: Dr Hiroshi Yaegashi, Department of Integrative Cancer Therapy and Urology, Kanazawa University Graduate School of Medical Science, 13-1 Takaramachi, Kanazawa, Ishikawa 920-8640, Japan

E-mail: hyae2002jp@yahoo.co.jp

Key words: metastatic urachal cancer, combination chemotherapy, gemcitabine, cisplatin, radiotherapy
Because of the silent nature of early lesions, their propensity for local growth and the tendency to metastasize late in the clinical course, a large proportion of patients with $\mathrm{UrC}$ present with disease at the pT3 stage: Local extension to the (a) bladder, (b) abdominal wall, (c) viscera other than the bladder, or higher (1).

UrC consisted of well differentiated tumors, and non-involvement of adjacent organs and the peritoneum indicates better prognosis when treated operatively (3), however, there have been lack of evidence for advanced UrC. Furthermore, a recurrent rate is very high in patient with advanced $\mathrm{UrC}$ even if treated operatively.

Patients with metastatic or recurrent UrC have extreme poor prognosis because there is currently no established standard regimen in chemotherapy or radiation protocol for these patients. A median survival time of patients with metastatic $\mathrm{UrC}$ is reported as up to 1.3 years at best (4). Here, we report a patient with metastatic $\mathrm{UrC}$ who achieved a comparatively long-term survival with gemcitabine (GEM)/cisplatin (CDDP) combination chemotherapy and radiotherapy.

\section{Case report}

A 55-year-old female reported to our department with right lower abdominal pain in March 2013. The patient did not present with any other diseases and urinary symptoms. A contrast-enhanced computed tomography (CT) examination at the first visit showed a tumor extended along the course of the urachus (Fig 1A-C). Peritoneal dissemination spread to the surface of the liver (Fig. 1D). Both serum carcinoembryonic antigen (CEA) and carbohydrate antigen (CA) 125 were present in high levels, with values of $294 \mathrm{ng} / \mathrm{ml}$ and $92 \mathrm{U} / \mathrm{ml}$, respectively. The patient immediately underwent transurethral biopsy of the urachal tumor, which revealed urachal adenocarcinoma (Fig. 2). She was accordingly diagnosed with Sheldon's stage IVB. As she did not previously have a history of urachal cancer, the disease was not recurrent and was considered primary.

Because surgical resection for primary site is not curative for this stage, she was eventually administered systemic 

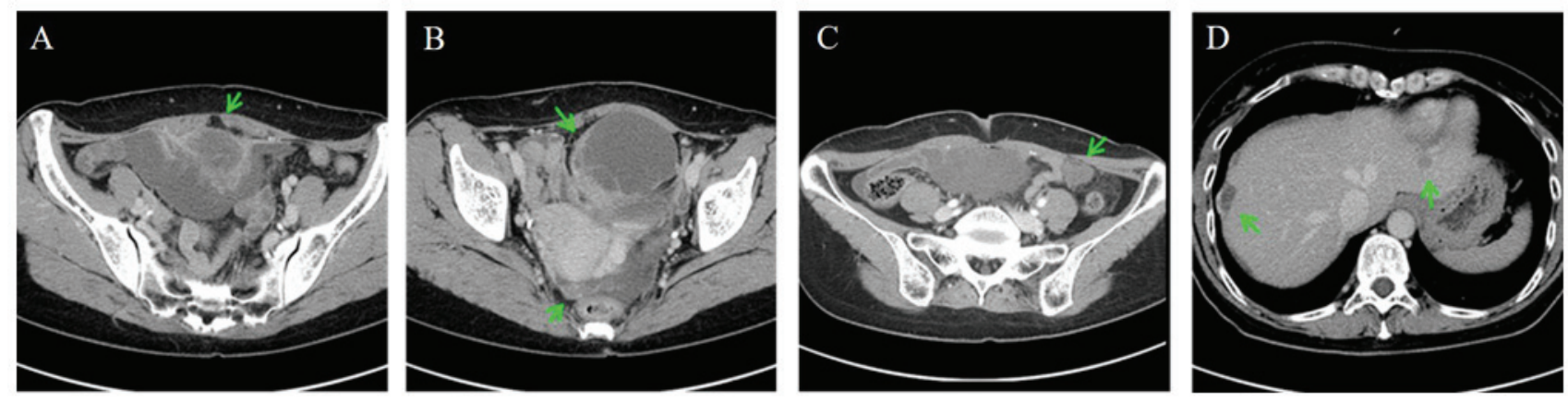

Figure 1. Contrast-enhanced computed tomography examination at the first visit. (A and B) A tumor extended along the course of urachus. (C) Direct invasion into rectus abdominis muscle is detected. (D) Multiple peritoneal dissemination spread to the surface of the liver is detected (indicated by arrows).

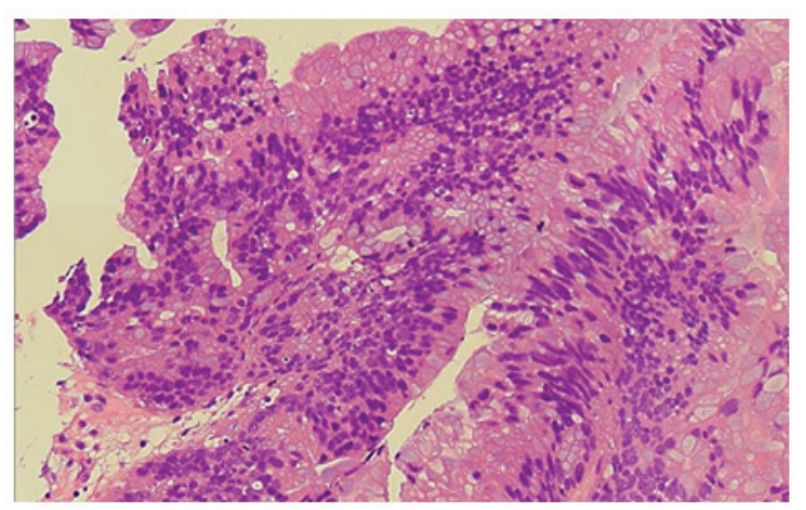

Figure 2. Well to moderately differentiated enteric mutinous adenocarcinoma is revealed by hematoxylin and eosin staining. Invasion into the muscle layer was partly detected. Hematoxylin and eosin staining; magnification, x400.

chemotherapy since April 2013 (repeated every 4 weeks) with GEM $\left(1,000 \mathrm{mg} / \mathrm{m}^{2} /\right.$ day $)$ on day $1,8,15$, and CDDP (70 $\mathrm{mg} / \mathrm{m}^{2} /$ day) on day 2 . The CT examination after first course of chemotherapy showed exacerbation of her right hydronephrosis and renal function declined at the end of the first course, we inserted an indwelling right ureteral stent for right hydronephrosis.

After confirming improved renal function, we adopted the strategy of GEM plus split-dose CDDP method (GEM: $1,000 \mathrm{mg} / \mathrm{m}^{2} /$ day, day $1,8,15, \mathrm{CDDP}: 35 \mathrm{mg} / \mathrm{m}^{2} /$ day, day 2 , day 9 , repeated every 4 weeks). She completed the chemotherapy without any change to her renal function through course 6. Serum levels of CEA and CA 125 declined to $37 \mathrm{ng} / \mathrm{ml}$ and $29 \mathrm{U} / \mathrm{ml}$, respectively, and radiological examination showed disease stability. Chemotherapy was discontinued once, owing to the patient's limited physical strength. Though CT examination at 10 months after initial diagnosis revealed disease stability, examination using 18F-FDG positron emission tomography at 11 months after initial diagnosis led us to suspect viable cells at a primary site; therefore, we conducted external beam radiation therapy (EBRT, 50.4 Gy, 28 fractions, Fig. 3A and B).

Because serum CEA and CA 125 gradually increased and we observed progression of multiple peritoneal metastases after EBRT at 19 months after initial diagnosis, we administered additional chemotherapy with GEM plus split-dose CDDP method. However, she did not complete chemotherapy due to hematological toxicity and exhibited a decline in her status. Her general condition gradually deteriorated, and she died of urachal adenocarcinoma 23 months after initial diagnosis.

\section{Discussion}

The therapeutic strategy for metastatic UrC has not yet been established because of its rare occurrence. A review with meta-analysis conducted on 1,010 cases of urachal cancer suggested the superiority of 5-FU-containing chemotherapy regimens to cisplatin-based chemotherapy regimens. However, the authors indicated that their combination seemed to provide the strongest anti-tumor effect (5). Furthermore, some reports have confirmed the efficacy of combination chemotherapy for metastatic UrC.

Incidentally, gemcitabine, which is an analog of deoxycytidine, is also an inhibitor of DNA synthesis (6). The combination chemotherapy with this drug is still considered to be standard induction chemotherapy for many types of advanced cancer such as pancreatic cancer (7), biliary tract cancer (8), and urothelial carcinoma of the bladder (9). Regarding metastatic $\mathrm{UrC}$, some reports have detailed the efficacy of combination chemotherapy with GEM, and some cases achieved complete or partial response in several case reports or series (10-12). Urologists have an expertise in this treatment method because GEM/CDDP combination chemotherapy is now considered the gold standard for both advanced upper tract urothelial cancer and bladder cancer.

In terms of histological similarity of UrC to colon cancer, reports of FOLFOX (oxaliplatin, 5-FU, and leukovolin) regimen for metastatic $\mathrm{UrC}$ have been found on occasion. Nevertheless, they are all in the case series $(13,14)$. Similarly, although CPT-11/TS-1 combination chemotherapy is considered as a salvage or adjuvant therapy (15), and TS-1/CDDP combination chemotherapy for UrC with multiple lung metastases (16) has been reported; they are only limited cases.

With respect to radiotherapy, the effectiveness of EBRT for bone metastases in a metastatic UrC case to relieve pain has been reported (10). Although EBRT for primary site has been reported and has showed a certain therapeutic efficacy (17), it remains in the case series.

Regarding therapeutic effect, Hasegawa et al (18) recommended measurements of serum CEA and CA 19-9 because their value appears to reflect the progression of $\mathrm{UrC}$. 

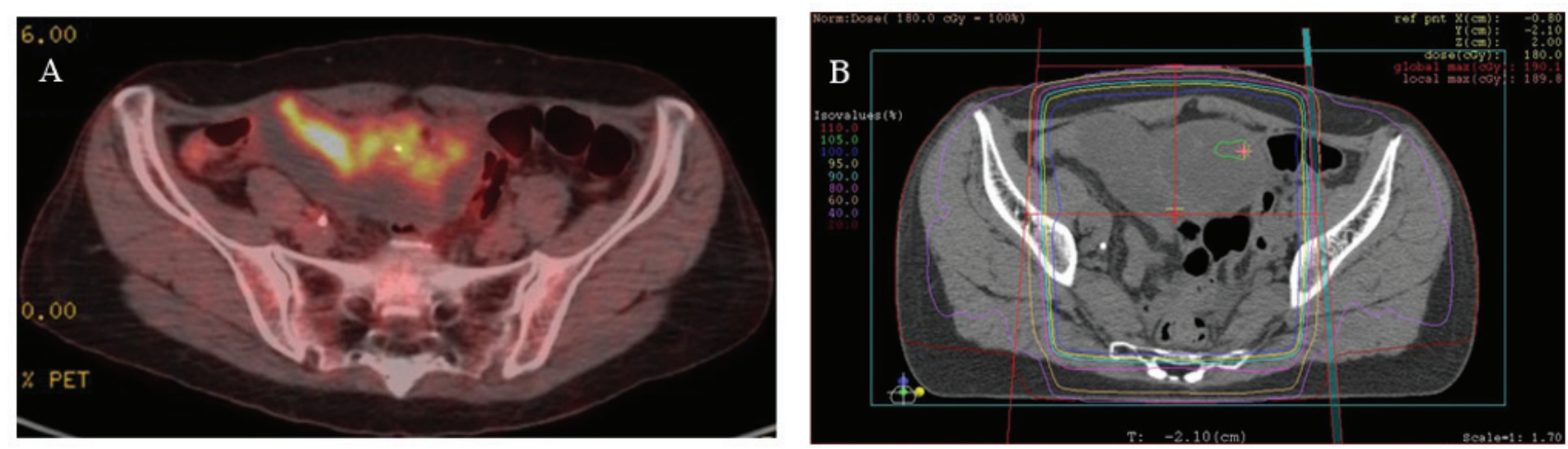

Figure 3. (A) 18F-fluorodeoxyglucose positron emission tomography at a period of 11 months after initial diagnosis suspected of viable cells at a primary site. The dose-distribution of external beam radiotherapy is as shown in the image. (B) The isodose curve for urachal tumor is drawn.

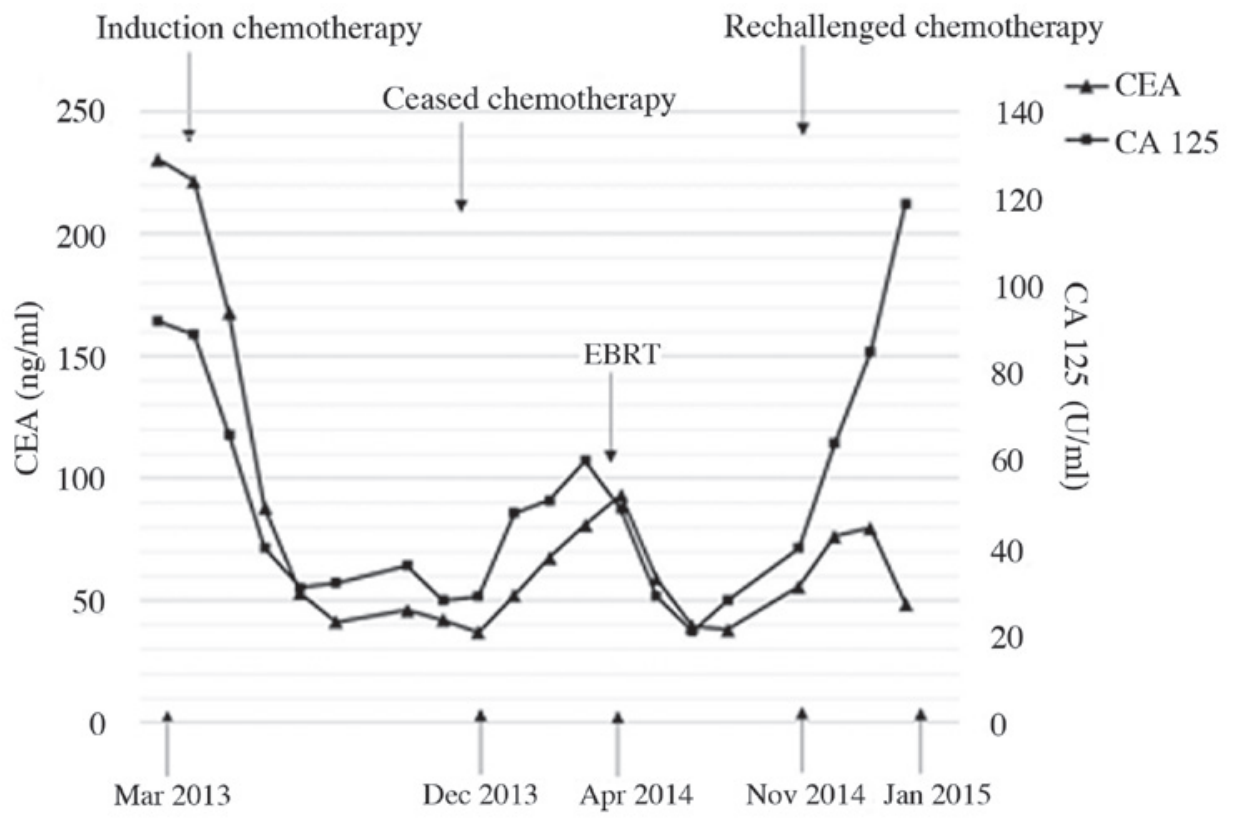

Figure 4. Transitive graph of serum CEA and CA 125 with clinical course. CEA, carcinoembryonic antigen; CA 125, carbohydrate antigen 125; EBRT, external beam radiation therapy.

Zong and Chen (19) also indicated efficacy of serum tumor markers such as CA 724, CA 125, CA 19-9, and CEA for a case report of urachal cancer with repeated relapses.

Based on these facts, we measured serum CEA, CA 19-9 and CA 125 at initial diagnosis as well and found an increase in CEA and CA 125. Because serum CEA and CA 125 reflected some improved therapeutic effect in our present case (Fig. 4), the measurement and follow-up of such tumor markers should be necessary and standard during treatment.

In conclusion, we observed a case of metastatic UrC treated with combination chemotherapy and EBRT. The evaluation of tumor markers such as CEA and CA 125 appear to be effective in predicting therapeutic response. Further investigation is warranted to improve survival rates in metastatic UrC.

\section{Acknowledgements}

Not applicable.

\section{Funding}

No funding was received.

\section{Availability of data and materials}

The datasets used and/or analyzed during the current study are available from the corresponding author on reasonable request.

\section{Authors' contributions}

HY, KI and AM designed the study and drafted the manuscript. TN, KS and YK made substantial contributions to the study conception and design. HY also retrieved the pathology images. HY, SK, RN, TM, HI and KI reviewed the patient's history, and clinical and imaging data. AM supervised the entire project. HY and KI critically revised the manuscript. All the authors have read and approved the final version of this manuscript. 


\section{Ethics approval and consent to participate}

The Ethics Committee of Kanazawa University waived the requirement for ethical approval and patient consent due to the retrospective nature of the report and as the patients' relatives were unable to be reached.

\section{Patient consent for publication}

Not applicable.

\section{Competing interests}

The authors declare that they have no competing interests.

\section{References}

1. Gopalan A, Sharp DS, Fine SW, Tickoo SK, Herr HW, Reuter VE and Olgac S: Urachal carcinoma: A clinicopathologic analysis of 24 cases with outcome correlation. Am J Surg Pathol 33: 659-668, 2009

2. Johnson DE, Hodge GB, Abdul-Karim FW and Ayala AG: Urachal carcinoma. Urology 26: 218-221, 1985.

3. Pinthus JH, Haddad R, Trachtenberg J, Holowaty E, Bowler J, Herzenberg AM, Jewett M and Fleshner NE: Population based survival data on urachal tumors. J Urol 175: 2042-2047; discussion 2047, 2006.

4. Yanagihara Y, Tanji N, Miura N, Shirato A, Nishimura K, Fukumoto T, Azuma K, Miyauchi Y, Kikugawa T and Yokoyama M: Modified FOLFOX6 chemotherapy in patients with metastatic urachal cancer. Chemotherapy 59: 402-406, 2013.

5. Szarvas T, Módos O, Niedworok C, Reis H, Szendröi A, Szász MA and Nyirády P: Clinical, prognostic, and therapeutic aspects of urachal carcinoma-A comprehensive review with meta-analysis of 1,010 cases. Urol Oncol 34: 388-398, 2016.

6. Plunkett W, Huang P, Xu YZ, Heinemann V, Grunewald R and Gandhi V: Gemcitabine: Metabolism, mechanisms of action, and self-potentiation. Semin Oncol 22: 3-10, 1995.
7. Kamisawa T, Wood LD, Itoi T and Takaori K: Pancreatic cancer. Lancet 388: 73-85, 2016.

8. Woo SM, Lee WJ, Kim JH, Kim DH, Han SS, Park SJ, Kim TH, Lee JH, Koh YH and Hong EK: Gemcitabine plus cisplatin versus capecitabine plus cisplatin as first-line chemotherapy for advanced biliary tract cancer: A retrospective cohort study. Chemotherapy 59: 232-238, 2013.

9. El Karak F and Flechon A: Gemcitabine in bladder cancer. Expert Opin Pharmacother 8: 3251-3256, 2007.

10. Ebara S, Kobayashi Y, Sasaki K, Araki M, Sugimoto M, Wada K, Fujio K, Takamoto A, Watanabe T, Yanai H and Nasu Y: A case of metastatic urachal cancer including a neuroendocrine component treated with gemcitabine, cisplatin and paclitaxel combination chemotherapy. Acta Med Okayama 70: 223-227, 2016.

11. Yu B, Zhou J, Cai H, Xu T, Xu Z, Zou Q and Gu M: Neoadjuvant chemotherapy for primary adenocarcinomas of the urinary bladder: A single-site experience. BMC Urol 15: 3, 2015.

12. Miyata Y, Sagara Y, Matsuo T, Ohba K, Takahashi H, Sakai H and Kanetake $\mathrm{H}$ : Response of recurrent urachal cancer to gemcitabine and cisplatin therapy: A case report and literature review. Anticancer Res 31: 2335-2338, 2011.

13. Tran B and McKendrick J: Metastatic urachal cancer responding to FOLFOX chemotherapy. Can J Urol 17: 5120-5123, 2010.

14. Kikuchi M, Kamei S, Morirama Y, Tuchiya T, Miwa K, Yokoi S, Nakano M, Ehara H, Deguchi T and Hirose Y: Case of urachal cancer treated by neoadjuvant chemotherapy with FOLFOX4 (oxaliplatin, 5-FU and leukovolin). Hinyokika Kiyo 54: 557-559, 2008 (In Japanese).

15. Yazawa S, Kikuchi E, Takeda T, Matsumoto K, Miyajima A, Nakagawa K and Oya M: Surgical and chemotherapeutic options for urachal carcinoma: Report of ten cases and literature review. Urol Int 88: 209-214, 2012.

16. Yoshida Y, Yamanaka K, Ueda N, Hirai T, Kishikawa H, Nishimura K and Ichikawa Y: A case of urachal carcinoma with multiple lung metastases treated by TS-1/CDDP chemotherapy. Hinyokika Kiyo 60: 147-150, 2014 (In Japanese).

17. Tian J, Ma JH, Li CL and Xiao ZD: Urachal mass in adults: Clinical analysis of 33 cases. Zhonghua Yi Xue Za Zhi 88: 820-822, 2008 (In Chinese).

18. Hasegawa Y, Kato Y, Wakita T, Hayashi N and Tsukamoto K: Carcinoma of the urachus: A case report. Hinyokika Kiyo 51: 191-194, 2005 (In Japanese).

19. Zong L and Chen P: Surgical and chemotherapeutic experience regarding a urachal carcinoma with repeated relapse: Case report and literature review. World J Surg Oncol 11: 170, 2013. 